\title{
Neuroprotective Effects of Triterpene Glycosides from Glycine max against Glutamate Induced Toxicity in Primary Cultured Rat Cortical Cells
}

\author{
Hyung-In Moon * and Jai-Heon Lee
}

Department of Medicinal Biotechnology, College of Natural Resources and Life Science, Dong-A University, Busan 604-714, Korea; E-Mail: hoffmannu@nate.com

* Author to whom correspondence should be addressed; E-Mail: himun68@dau.ac.kr; Tel.: +82-51-200-7562; Fax: +82-51-200-6195.

Received: 7 June 2012; in revised form: 16 July 2012 / Accepted: 23 July 2012 /

Published: 2 August 2012

\begin{abstract}
To examine the neuroprotective effects of Glycine max, we tested its protection against the glutamate-induced toxicity in primary cortical cultured neurons. In order to clarify the neuroprotective mechanism(s) of this observed effect, isolation was performed to seek and identify active fractions and components. From such fractionation, two triterpene glycosides, 3- $O$-[ $\alpha$-L-rhamnopyranosyl(1-2)- $\beta$-D-glucopyranosyl(1-2)- $\beta$-Dglucuronopyranosyl]olean-12-en-3 $\beta, 22 \beta, 24$-triol (1) and 3- $O$-[ $\beta$-D-glucopyranosyl(1-2)- $\beta$ D-galactopyranosyl(1-2)- $\beta$-D-glucuronopyranosyl]olean-12-en-3 $\beta, 22 \beta, 24$-triol (2) were isolated with the methanol extracts with of air-dried Glycine max. Among these compounds, compound $\mathbf{2}$ exhibited significant neuroprotective activities against glutamate-induced toxicity, exhibiting cell viability of about $50 \%$ at concentrations ranging from $0.1 \mu \mathrm{M}$ to $10 \mu \mathrm{M}$. Therefore, the neuroprotective effect of Glycine max might be due to the inhibition of glutamate-induced toxicity by triterpene glycosides.
\end{abstract}

Keywords: Glycine max; glutamate-induced toxicity; neuroprotective; triterpene glycosides

\section{Introduction}

Phytochemical compounds from grains are abundant in the human diet, particularly in fruit, vegetables and legumes, which have been consistently associated with a decreased risk of nutritional disease. They constitute one of the most abundant groups of natural metabolites and are now 
recognized for their important contribution to both health (and diet) effects in humans and animals. Epidemiological studies have consistently shown that regular consumption of fruits, vegetables, whole grains, and other plant foods is associated with reduced risk of developing chronic diseases, such as cancer and cardiovascular disease [1]. Legumes, especially Glycine maxs are widely consumed in the world, and are a staple food in Korea as well as being a major source for protein, energy, vitamins, and minerals. The phytochemicals of Glycine maxs, including phenolic compounds, phytic acid, triterpene glycosides, and phytosterols, may be responsible for their anticancer activity [2]. It was reported that Glycine maxs reduced azoxymethaneinduced colon cancer in a rat model [3]. Legume tannins are 15-30 times more effective at quenching peroxyl radicals than simple phenolics, thus they are potential biological antioxidants [4]. Although biological activity has been discovered from Glycine max, it has not been fully characterized.

Alzheimer's disease (AD) is an age-related neurodegenerative disease that affects approximately 24 million people worldwide [5]. Neuronal death is an important feature of both acute and chronic neurodegenerative diseases. $\mathrm{AD}$ is associated with the accumulation of L-glutamate (Glu) deposits in senile plaques and neurofibrillary tangle lesions in specific areas of brain. Glutamate, a major excitatory amino acid neurotransmitter in the central nervous system (CNS), involved in fast synaptic transmission, neuronal plasticity, outgrowth and survival, memory, learning and behavior. Glutamate also plays an important role in microglial neurotoxicity in AD. Activated microglia produce large amounts of glutamate, which induces excitotoxicity via $N$-methyl-D-aspartate (NMDA) receptor signaling [6]. Activated microglia release large amounts of glutamate through upregulation of glutaminase expression and induce excitoneurotoxicity through NMDA receptor signaling [7]. Apart from the physiological role of glutamate, excessive activation of its receptors can also evoke neuronal dysfunction and even damage or death. Glutamate-mediated neurotoxicity appears to play a crucial role in several neuropathological disorders, particularly in Alzheimer's disease, Parkinson's disease, epilepsy and ischemic stroke [8]. Thus, neuroprotection against glutamate-induced toxicity has been a therapeutic strategy for preventing and/or treating both acute and chronic forms of neurodegeneration [9].

In the context of our natural drugs discovery program dealing with the development of new potential neuroprotection agents, we have examined the isolation of two compounds, $3-O-[\alpha-\mathrm{L}-$ rhamnopyranosyl(1-2)- $\beta$-D-glucopyranosyl(1-2)- $\beta$-D-glucuronopyranosyl]olean-12-en-3 $\beta, 22 \beta, 24$-triol (1) and 3-O-[ $\beta$-D-glucopyranosyl(1-2)- $\beta$-D-galactopyranosyl(1-2)- $\beta$-D-glucuronopyranosyl]olean-12-en$3 \beta, 22 \beta, 24$-triol (2) as leads for novel glutamate-induced toxicity inhibitors.

\section{Results and Discussion}

It was found that the methanol extract of Glycine max might inhibit glutamate-induced toxicity in primary cultured rat cortical cells. In order to clarify the neuroprotective components of Glycine max, as a part of continued study of the neuroprotection effects of Glycine max, isolation was performed to seek active fractions and components. After solvent fractionation, the inhibiting effects of various fractions on neuroprotective activity were compared and the ethyl acetate fraction of Glycine max was found to inhibit this activity in a dose-dependent manner in the assay system using glutamate-induced toxicity in primary cultured rat cortical cells. To clarify the active compounds of Glycine max, the effects of the major compounds from Glycine $\max$ on neuroprotective activity were examined. 
Isolation was further performed to yield a single compound. Bioactive triterpene glycosides derivatives were isolated from the ethyl acetate fraction by repeated chromatography and recrystallization. The ethyl acetate fraction (35 g) was further purified by silica gel chromatography (230-400 mesh, $500 \times 75 \mathrm{~mm}$ ) and four subfractions (BB-1-4) whose main active subfraction (BB-3; $5.8 \mathrm{~g}$ ) was purified by VLC and HPLC, and compound fractions $1(7.2 \mathrm{mg})$ and $2(4.7 \mathrm{mg})$ were obtained from hexane/ethyl acetate (20:3) elution. Then, the subfraction $\mathrm{CH}_{2} \mathrm{Cl}_{2} / \mathrm{MeOH}(10: 1)$ was further purified on preparative HPLC using the $250 \times 22 \mathrm{~mm}$ i.d., $5 \mu \mathrm{m}$, Agilent $\mathrm{C}_{18}$ preparative HPLC column, which was eluted isocratically with $20 \%$ acetonitrile in water at a flow rate of $8.0 \mathrm{~mL} / \mathrm{min}$. Compounds 1 $(3.2 \mathrm{mg})$ and $2(2.1 \mathrm{mg})$ were obtained at retention times of 5.3 and $14.7 \mathrm{~min}$, respectively. Thin layer chromatography and preparative high-performance liquid chromatography methods of the $\mathrm{MeOH}$ extract of harvested Glycine $\max$ led to the isolation of two compounds. Compound $\mathbf{1}$ was identified as 3-O-[ $\alpha$-L-rhamnopyranosyl(1-2)- $\beta$-D-glucopyranosyl(1-2)- $\beta$-D-glucuronopyranosyl]olean12-en-3 $\beta, 22 \beta, 24$-triol. Compound $\mathbf{2}$ was identified as 3-O-[ $\beta$-D-glucopyranosyl(1-2)- $\beta$-Dgalactopyranosyl(1-2)- $\beta$-D-glucuronopyranosyl]olean-12-en-3 $\beta, 22 \beta, 24$-triol (Figure 1). The compounds have previously been reported as antiproliferative and antioxidative activity from the Glycine max seed coats by Dong et al. [10]. In this study, we found that methanol extract of Glycine max might inhibit glutamate-induced toxicity in primary cultured rat cortical cells. In order to clarify the neuroprotective components of Glycine max, as part of a continued study of neuroprotection effects of Glycine max, isolation was performed to seek active fractions and components. After solvent fractionation, we compared the inhibiting effects of various fractions on neurotoxicity activity. The BB-3 fraction of Glycine $\max$ was found to inhibit the activity in a dose-dependent manner in glutamate-induced toxicity assay using primary cultured rat cortical cells (Table 1). To clarify the active substances of Glycine max, we examined the effects of the major isolated compounds from BB-3 fraction on neuroprotective activity. Isolation was further performed to yield single compounds.

Figure 1. Isolated compounds structure from Glycine max.

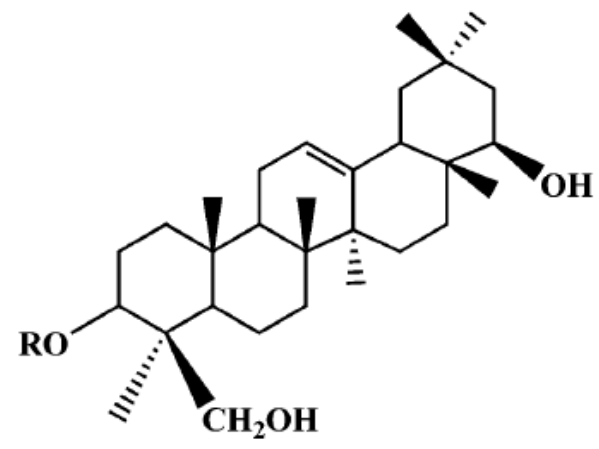

1

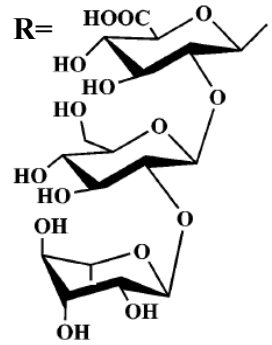

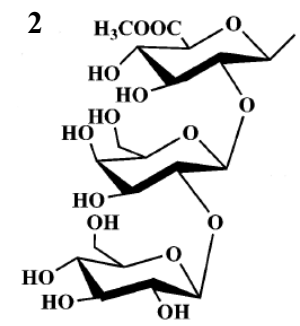


Table 1. Neuroprotective effects of extract and fractions from Glycine max against glutamate induced toxicity in primary cultured rat cortical cells ${ }^{\text {a }}$.

\begin{tabular}{|c|c|c|c|}
\hline \multirow{2}{*}{ Dose } & \multicolumn{3}{|c|}{ Cell Viability $^{b, d}(\%)$} \\
\hline & $5 \mu \mathrm{g} / \mathrm{mL}$ & $25 \mu \mathrm{g} / \mathrm{mL}$ & $100 \mu \mathrm{g} / \mathrm{mL}$ \\
\hline Control $^{\mathrm{c}}$ & & 100 & \\
\hline Glutamate-treated $^{\mathrm{c}, \mathrm{e}}$ & & 0 & \\
\hline $\mathrm{MeOH}$ extracts & $13.4 \pm 0.3$ & $38.5 \pm 3.2 *$ & $59.0 \pm 3.6 * *$ \\
\hline Chloroform fraction & - & - & $12.3 \pm 1.3$ \\
\hline Ethyl acetate fraction & - & $21.4 \pm 6.3 *$ & $55.3 \pm 5.7 * *$ \\
\hline n-butanol fraction & - & - & $5.8 \pm 1.3$ \\
\hline BB-1 subfraction & - & - & - \\
\hline BB-2 subfraction & - & - & - \\
\hline BB-3 subfraction & $15.6 \pm 3.6^{*}$ & $36.7 \pm 3.7 *$ & $43.5 \pm 2.6 * *$ \\
\hline BB-4 subfraction & - & - & $26.7 \pm 8.9$ \\
\hline BB-5 subfraction & - & - & - \\
\hline
\end{tabular}

${ }^{a}$ Rat cortical cell cultures were incubated with test compounds for $1 \mathrm{~h}$. The cultures were then exposed to $100 \mu \mathrm{M}$ glutamate for $24 \mathrm{~h}$. After the incubation, the cultures were assessed for the extent of neuronal damage; ${ }^{b}$ Cell viability was measured by the LDH assay; ${ }^{\mathrm{c}} \mathrm{LDH}$ released from control $(0.1 \%$ DMSO concentration in culture) and glutamate-treated cultures were $11.7 \pm 1.3$ and $47.9 \pm 4.0$ units $/ \mathrm{mL}$, respectively; ${ }^{\mathrm{d}}$ Cell viability was calculated as $100 \times(\mathrm{LDH}$ released from glutamate-treated-LDH released from glutamate + test compound-treated)/(LDH released from glutamate-treated-LDH released from control). The values shown are the mean \pm STD of three experiments (3-4 cultures per experiment). Results differ significantly from the glutamate-treated: $* p<0.05,{ }^{* *} p<0.01, * * * p<0.001{ }^{*}{ }^{\mathrm{e}}$ Glutamate-treated value differed significantly from the untreated control at the level of $p<0.001$.

Two known compounds were isolated from BB-3 fraction by repeated VLC and HPLC. To investigate and compare the neuroprotective activities of these compounds isolated from the methanol extracts of Glycine max, the activities of compounds $\mathbf{1}$ and $\mathbf{2}$ were evaluated in glutamate-injured primary cultured rat cortical cells at concentrations ranging from 0.1 to $10 \mu \mathrm{M}$ (Table 2). It is notable that the neuroprotective activities of compound 2 was comparable to those of MK-801, APV and CNOX, and, only compound $\mathbf{2}$ displayed an activity which significantly attenuated glutamate-induced toxicity at concentrations ranging from 0.1 to $10 \mu \mathrm{M}$ and exhibited cell viabilities of $50-70 \%$.

\section{Experimental Section}

\subsection{Plant Material}

Glycine max cultivars (Black beans) were purchased from the local market in Hwa Sung, Kunggi-Do, Korea. The botanical identification was made by Hyung-In Moon in Dong-A University (Busan, Korea). Voucher herbarium specimens were deposited with the reference number (DA-002-003) in the Herbarium of the Department of Medicinal Biotechnology, College of Natural Resources and Life Science, Dong-A University. 
Table 2. Neuroprotective effects of triterpene glycosides from Glycine max against glutam ate induced toxicity in primary cultured rat cortical cells ${ }^{\text {a }}$.

\begin{tabular}{lccc}
\hline \multirow{2}{*}{ Dose } & \multicolumn{3}{c}{ Cell Viability $^{\mathbf{b , d}} \mathbf{( \% )}$} \\
\cline { 2 - 4 } & $\mathbf{0 . 1} \boldsymbol{\mu} \mathbf{M}$ & $\mathbf{1} \boldsymbol{\mu M}$ & $\mathbf{1 0} \boldsymbol{\mu M}$ \\
\hline Control $^{\mathrm{c}}$ & \multicolumn{3}{c}{100} \\
Glutamate-treated $^{\mathrm{c}, \mathrm{e}}$ & & 0 & \\
$\mathbf{1}$ & $14.2 \pm 0.3$ & $16.7 \pm 1.2$ & $21.4 \pm 5.6$ \\
$\mathbf{2}$ & $16.7 \pm 1.5 *$ & $39.2 \pm 1.5 * *$ & $71.5 \pm 6.8 * * *$ \\
APV $^{\mathrm{f}}$ & $11.5 \pm 1.4$ & $26.5 \pm 2.3 *$ & $42.5 \pm 3.7 *$ \\
MK-801 $^{\mathrm{g}}$ & $51.4 \pm 4.6^{* *}$ & $63.5 \pm 5.8^{* * *}$ & $78.4 \pm 2.0 * * *$ \\
$\mathrm{CNQX}^{\mathrm{h}}$ & $23.5 \pm 3.7 *$ & $44.8 \pm 3.5 *$ & $53.2 \pm 4.6 * * *$ \\
\hline
\end{tabular}

${ }^{\text {a }}$ Rat cortical cell cultures were incubated with test compounds for $1 \mathrm{~h}$. The cultures were then exposed to $100 \mu \mathrm{M}$ glutamate for $24 \mathrm{~h}$. After the incubation, the cultures were assessed for the extent of neuronal damage; ${ }^{\mathrm{b}}$ Cell viability was measured by the LDH assay; ${ }^{\mathrm{c}} \mathrm{LDH}$ released from control ( $0.1 \%$ DMSO concentration in culture) and glutamate-treated cultures were $11.7 \pm 1.3$ and $47.9 \pm 4.0$ units $/ \mathrm{mL}$, respectively; ${ }^{\mathrm{d}}$ Cell viability was calculated as $100 \times(\mathrm{LDH}$ released from glutamate-treated-LDH released from glutamate + test compound-treated)/(LDH released from glutamate-treated-LDH released from control). The values shown are the mean \pm STD of three experiments (3-4 cultures per experiment). Results differ significantly from the glutamate-treated: $* p<0.05, * * p<0.01, * * * p<0.001 ;{ }^{\mathrm{e}}$ Glutamate-treated value differed significantly from the untreated control at the level of $p<0.001$; ${ }^{\mathrm{f}}$ APV: DL-2-amino-5-phosphonovaleric acid, a competitive NMDA receptor antagonist; ${ }^{\mathrm{g}} \mathrm{MK}-801$ : dizocilpine maleate, a noncompetitive NMDA receptor antagonist; ${ }^{\mathrm{h}} \mathrm{CNQX}: 6^{-}$cyan $^{\circ}$-7-nitroquinoxaline-2,3-dione, non-NMDA receptor antagonist.

\subsection{Extraction and Isolation of Compounds}

The Glycine maxs $(1 \mathrm{~kg})$ were extracted by percolation in $95 \%$ methanol $(\mathrm{MeOH} ; 2000 \mathrm{~mL})$ at room temperature for 1 week and filtered. The combined $\mathrm{MeOH}$ extracts $(212 \mathrm{~g})$ were concentrated under reduced pressure at a temperature not exceeding $35^{\circ} \mathrm{C}$. The residue was re-percolated again. This process was repeated four times. Water $(500 \mathrm{~mL})$ was added and the resultant mixture successively extracted with chloroform, ethyl acetate, and $n$-butanol, respectively $\mathrm{The}^{\mathrm{IC}} \mathrm{C}_{50}$ value for a neuroprotective effect of each fraction was found only for the ethyl acetate extracts fraction. The ethyl acetate fraction which possessed the potential neuroprotective effect $\left(\mathrm{IC}_{50}=81.2 \mu \mathrm{g} / \mathrm{mL}\right)$ was BB-subfraction chromatographed on silica gel using chloroform and methanol. The ethyl acetate fraction (30 g) was applied to a silica gel column and eluted with chloroform-methanol mixtures of increasing polarity $(95: 5(500 \mathrm{~mL}), 9: 1(500 \mathrm{~mL}), 4: 1(500 \mathrm{~mL}), 7: 3(500 \mathrm{~mL}), 1: 1(500 \mathrm{~mL}))$ to give four subfractions (BB-1-4) whose main active subfraction (BB-3; $5.8 \mathrm{~g}$ ) was purified by VLC and HPLC. This compounds with isolate methods were modified to Dong et al. [8].

\subsection{Neuroprotective Activity Testing}

Primary cultures of mixed cortical cells containing both neurons and glia were prepared from 17-19-day-old fetal rats (Sprague-Dawley) as described previously [11]. Cultures were allowed to mature for at least 2 weeks before being used for experiments. Test fraction and compounds were dissolved in DMSO (final concentration in culture, $0.1 \%$ ). Cortical cell cultures were washed with 
DMEM and incubated with test compounds for $1 \mathrm{~h}$. The cultures were then exposed to $100 \mu \mathrm{M}$ glutamate and maintained for $24 \mathrm{~h}$. After the incubation, the cultures were assessed for the extent of neuronal damage by measuring the efflux of LDH (lactic dehydrogenase) which reflects the integrity of cellular membrane. The results are expressed as means \pm standard errors (S.E.). The data were statistically analyzed by one way ANOVA. Differences with $p<0.05$ were considered significant.

\section{Conclusions}

Glutamate-induced neurotoxicity has been implicated in the neuronal cell death of neurological disorders such as ischemia, trauma, seizures, and Alzheimer's disease [6]. During our search for potential natural products against glutamate-induced neurotoxicity, we have discovered that the ethyl acetate sub-fraction (BB-3 fraction) of Glycine max showed significant protective activity. The neuroprotective activities of the triterpene glycosides glycosides were evaluated by the MTT assay measuring the viability in primary cultured rat cortical cells after glutamate insult. Among these two triterpene glycosides glycosides, compound $\mathbf{2}$ significantly attenuated neuronal cell death induced by glutamate in cultures. It was found to exhibit the most potent neuroprotective activity at a concentration of $10 \mu \mathrm{M}$. Our result supported that compound 2 was isolated from the Glycine max possess neuroprotective activities at low concentration and in a dose-dependent manner in primary cultured rat cortical cells. At present, the cellular and molecular mechanisms that underlie the action of compound $\mathbf{2}$ are not fully understood. However, future research data will show whether compound $\mathbf{2}$ acts on glutamate receptors, especially NMDA receptors by the following proposed pathway: (i) a protection of cortical cultures, (ii) selective protection in NMDA-induced neurotoxicity; (iii) blocking $\mathrm{Ca}^{2+}$ influx in glutamate insult; (iv) a reduction in NO and cellular peroxide production; (v) a reduction in GSH depletion and membrane lipid peroxidation. On the basis of our present study, the protective effect of compound 2 from Glycine max may provide some ideas for preventing and/or treating the neurodegenerative diseases.

\section{Acknowledgments}

This work was supported by the R\&D program of MKE/KEIT.(10040391, Development of Functional Food Materials and Device for Prevention of Aging-associated Muscle Function Decrease).

\section{Conflict of Interest}

The authors declare no conflict of interest.

\section{References}

1. Aparicio-Fernández, X.; García-Gasca, T.; Yousef, G.G.; Lila, M.A.; González de Mejia, E.; Loarca-Pina, G. Chemopreventive activity of polyphenolics from black Jamapa bean (Phaseolus vulgaris L.) on HeLa and HaCaT cells. J. Agric. Food Chem. 2006, 54, 2116-2122.

2. Iriti, M.; di Maro, A.; Bernasconi, S.; Burlini, N.; Simonetti, P.; Picchi, V.; Panigada, C.; Gerosa, G.; Parente, A.; Faoro, F. Nutritional traits of bean (Phaseolus vulgaris) seeds from plants chronically exposed to ozone pollution. J. Agric. Food Chem. 2009, 57, 201-208. 
3. Hangen, L.; Bennink, M.R. Consumption of black beans and navy beans (Phaseolus vulgaris) reduced azoxymethane-induced colon cancer in rats. Nutr. Cancer 2002, 44, 60-65.

4. Duodu, K.G.; Nunes, A.; Delgadillo, I.; Parker, M.L.; Mills, E.N.C.; Belton, P.S. Effect of grain structure and cooking on sorghum and maize in vitro protein digestibility. J. Cereal Sci. 2002, 35, 161-174.

5. Wang, R.; Zhou, J.; Tang, X.C. Tacrine attenuates hydrogen peroxideinduced apoptosis by regulating expression of apoptosisrelated genes in rat PC12 cells. Mol. Brain Res. 2002, 107, 1-8.

6. Sucher, N.J.; Awobuluyi, M.; Choi, Y.B.; Lipton, S.A. NMDA receptors: From genes to channels. Trends Pharmacol. Sci. 1996, 17, 348-355.

7. Mizuno, T. The biphasic role of microglia in Alzheimer's disease. Int. J. Alzheimer's Dis. 2012, doi:10.1155/2012/737846.

8. Koo, K.A.; Kim, S.H.; Oh, T.H.; Kim, Y.C. Acteoside and its aglycones protect primary cultures of rat cortical cells from glutamate-induced excitotoxicity. Life Sci. 2006, 79, 709-716.

9. Trist, D.G. Excitatory aminoacid agonists and antagonists: Pharmacology and therapeutic applications. Pharm. Acta Helvetiae 2000, 74, 221-229.

10. Dong, M.; He, X.; Liu, R.H. Phytochemicals of black bean seed coats: Isolation, structure elucidation, and their antiproliferative and antioxidative activities. J. Agric. Food Chem. 2007, 55, 6044-6051.

11. Koo, K.A.; Lee, M.K.; Kim, S.H.; Jeong, E.J.; Kim, S.Y.; Oh, T.H.; Kim, Y.C. Pinusolide and 15-methoxypinusolidic acid attenuate the neurotoxic effect of staurosporine in primary cultures of rat cortical cells. Br. J. Pharmacol. 2007, 150, 65-71.

(C) 2012 by the authors; licensee MDPI, Basel, Switzerland. This article is an open access article distributed under the terms and conditions of the Creative Commons Attribution license (http://creativecommons.org/licenses/by/3.0/). 